PlastOx 2007 (2009) 23-31

(C) EDP Sciences, 2009

DOI: $10.1051 /$ ptox/2009003

\title{
Simulation multiéchelle : dislocations de l'échelle atomique à l'échelle continue
}

\author{
G. Monnet et S. Queyreau \\ EDF - R\&D, Département MMC, Avenue des Renardières, 77818 Moret sur Loing, France \\ e-mail : Ghiath.Monnet@edf.fr
}

\section{INTRODUCTION}

La théorie élastique des dislocations a été établie vers la fin des années soixante [1]. Depuis, les différents travaux ont exploré l'univers de l'organisation de dislocations et ont conduit à l'élaboration de plusieurs modèles phénoménologiques visant à interpréter les propriétés mécaniques, comme le durcissement, l'écrouissage, endommagement, etc. directement à partir des propriétés individuelles des dislocations.

Parallèlement aux efforts théoriques continus, l'arrivé des outils de calculs avec une puissance grandissante, a conduit à l'apparition des technique de simulations numériques appropriées pour l'étude de dislocations : ab-initio, Dynamique Moléculaire (DM), Dynamique de Dislocations (DD), méthode Champs de Phases, Eléments Finis (EF). Ces techniques ont fourni des résultats importants dans deux domaines. D'abord les simulations atomiques ont révolutionné notre vision relative aux propriétés de cœur des dislocations : étalement, dissociation, interaction avec défauts ponctuels, etc. Deuxièmement, les simulations de grandes populations de dislocations par DD ont révélé plusieurs aspects du comportement collectif : coefficients d'interaction, écrouissage, durcissement par précipitation, etc...

Chacune des méthodes de simulations donne ainsi accès à un ensemble d'informations sur les dislocations, caractéristique de son propre échelle spatio-temporelle. Très vite, on s'est rendu compte de deux problèmes. D'une part, les résultats d'une méthode ne sont pas toujours compatibles avec ceux d'une autre, par exemple la contradiction entre les structures de cœurs prédites par ab initio et DM dans le fer et, d'autre part, les résultats des simulations ne sont pas toujours en accord avec l'expérience, par exemple l'échec de prédiction de systèmes actifs dans les structure Cubique-Centrée (CC) et Hexagonale - Compacte (HC).

Face à ces difficultés, une nouvelle approche est en cours de développement et ayant pour but la construction d'une vision complète et cohérente impliquent toutes les méthodes de simulation numérique et restant en accord avec les résultats expérimentaux. Cette approche, appelée multiéchelle, nécessite, au moins, deux étapes de transition d'échelle ou d' «homogénéisation » : échelle atomique (DM) - mésoscopique (DD) et mésoscopique (DD) - macroscopique (EF).

Dans ce document, nous présentons cette approche à travers un exemple d'interactioon entre une dislocation coin et une cavité.

\section{SIMULATION DE DYNAMIQUE MOLÉCULAIRE : DISLOCATION À L'ÉCHELLE ATOMIQUE}

\subsection{Principe}

Le principe des simulations de type DM est basé sur l'utilisation d'un potentiel atomique empirique, qui permet de prédire le vecteur force appliquée sur un atome en fonction des positions des atomes voisins. A partir de ce vecteur, on déduit la vitesse et la direction du déplacement de l'atome afin de le ramener en position d'équilibre mécanique. Toutes les informations relatives au matériau sont exclusivement contenues dans ce potentiel, d'où le point «critique » de cette méthode : lisser ce potentiel de telle sorte que les propriétés recherchées sont bien reproduites. Ainsi, un potentiel « raisonnable » pour l'étude des défauts ponctuels n'est pas obligatoirement un potentiel fiable pour l'étude de dislocations : un atome 

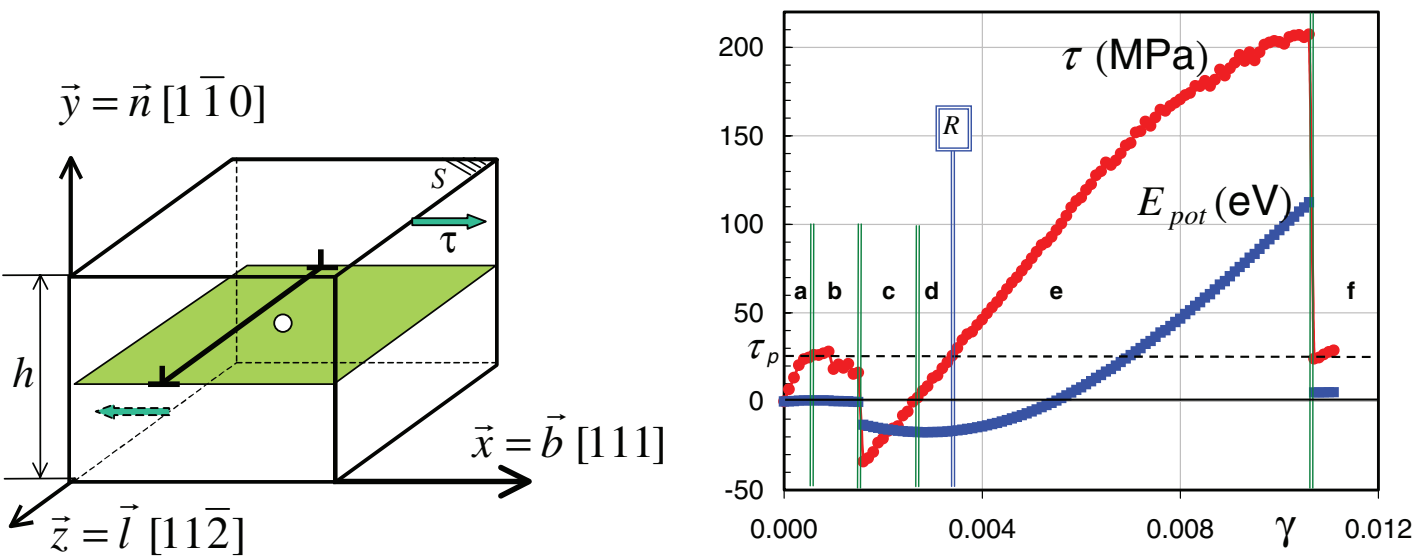

Figure 1. (À gauche) schéma représentant la boîte de simulation DM, (à droite) courbe de chargement avec l'évolution de l'énergie potentielle du système.

interstitiel provoque des distorsions cristallines à très courte portée (en $\left.1 / \mathrm{r}^{3}\right)$ alors que les dislocations entraînaient des champs de déformation à longue portée (en 1/r). Un des moyens les plus conservateurs pour vérifier la fiabilité du potentiel est de comparer la structure de cœur d'une dislocation prédite par ab initio et DM.

L'intérêt fondamental de cette technique de simulations est qu'elle soit la seule méthode prenant en compte, d'une façon explicite et transparent, l'activation thermique.

\subsection{Interaction entre dislocation coin et cavité}

La façon la plus simple de créer la dislocation vis est d'appliquer la solution élastique isotrope du champ de déformation de la dislocation et de positionner les atomes en conséquence. Pour la dislocation coin, il faut introduire un (ou plusieurs) demi-plan supplémentaire et laisser relaxer le system. L'interaction avec la cavité de $2 \mathrm{~nm}$ de taille a été simulée par Y. Ozetski et al. [2] utilisant le potentiel empirique d'Ackland [3] pour le fer, voir Fig. 1.

La simulation a été effectuée à très basse température et vitesse de déformation constante. La courbe de chargement montre que l'interaction passe par plusieurs régimes selon que la dislocation est attirée par la cavité (contrainte négative) ou épinglée par celle-ci (contrainte positive). Par ailleurs, l'énergie potentielle du système varie d'une façon comparable à celle de la contrainte. Afin d'arracher la dislocation à la cavité, il faut appliquer une contrainte poche de $210 \mathrm{MPa}$. Ces résultats sont obtenus directement sans faire appel à la théorie élastique des dislocations ni aux hypothèses des inclusions dans une matrice homogène, etc. Ils tiennent compte de l'anisotropie du matériau et de tous les détails de l'interaction (formation de marche de surface, montée de la dislocation par absorption de lacunes de la cavité, etc.) Néanmoins, ces résultats, par leur nature, comportent un aspect propre: toutes les valeurs obtenues sont dépendantes des dimensions de la boite de simulation ainsi que de la vitesse de déformation imposée. Or, d'une part ces conditions sont très loin des conditions expérimentales et, d'autre part, nous devons extraire des données caractéristiques de l'interaction, c'està-dire indépendantes des paramètres de dimensions.

\section{CONNEXION ENTRE L'ÉCHELLE ATOMIQUE ET L'ÉCHELLE CONTINUE}

Afin de traduire les résultats de la DM à l'échelle de la DD, nous devons gommer l'aspect atomique de ces résultats. Pour se faire, nous imaginons que la cavité est une sphère dans le milieu continu 


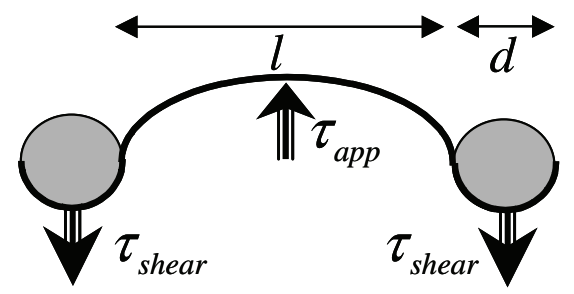

Figure 2. Équilibre mécanique autour de l'obstacle permettant de déduire $\tau_{\text {shear }}$.

caractérisée par une résistance à la pénétration des dislocations [4], $\tau_{\text {shear }}$. Une hypothèse d'équilibre mécanique peut toujours être émise : la force totale appliquée sur le segment de la dislocation entre cavités est égale à la force totale de résistance au cisaillement de l'obstacle, comme montré sur la Fig. 2. Par conséquent, nous pouvons déduire $\tau_{\text {shear }}$ de la contrainte appliquée $\tau_{a p p}$ qui, celle-ci, est bien fournie par la DM : $\tau_{\text {shear }}=l / d \tau_{\text {app }}$, où $l$ est la distance entre obstacles et $d$ la taille de l'obstacle.

A titre d'exemple, le cas de la cavité de $2 \mathrm{~nm}$ en interaction avec la dislocation coin, voir Fig. 1, conduit à une résistance de cisaillement de l'ordre de (42/2) fois la contrainte maximale, c'est-à-dire près de $4.4 \mathrm{GPa}$. Cette valeur est très élevée mais reste inférieur à la limite de cisaillement théorique.

La valeur de $\tau_{\text {shear }}$ ne dépend pas de l'espacement entre obstacle et caractérise la « force » intrinsèque de l'obstacle. Elle peut être donc associée à l'obstacle et utilisé directement dans la DD comme on va le voir.

\section{SIMULATION DE DYNAMIQUE DE DISLOCATIONS}

\subsection{Le code microMegas $(\mathrm{mM})$}

Le code microMegas (mM) [5,6] est développé actuellement en collaboration entre le Laboratoire d'Etude des Microstructures, unité mixte CNRS-ONERA à Châtillon, et le Département MMC du Centre d'EdF R\&D des Renardières. Il fait l'objet d'une licence Free Software protégée par un CopyRight GNU GENERAL PUBLIC LICENSE, Version 2, June 1991 Licence Open Source.

Le code $\mathrm{mM}$ a été initialement développé pour l'étude de la plasticité dans les matériaux de structure CFC comme l'aluminium et du cuivre. L'application de la DD au cas de la structure Hexagonale Compacte (HC) et Cubique Centrée (CC) a été entreprise à l'initiative de l'EDF [7]. Actuellement, $\mathrm{mM}$ est compatible avec les structures suivantes : Cubique Simple (CS), CFC, HC, CC et l'olivine (céramique).

\subsection{Principes des simulations}

La présence d'une dislocation dans un cristal conduit à l'augmentation significative de son énergie de Gibbs. Les dislocations sont, par conséquent, thermodynamiquement instables (hors d'équilibre) quelle que soit la température. La difficulté fondamentale dans la prédiction du comportement d'une dislocation réside dans le fait que l'énergie même d'un segment de longueur infinitésimale n'est pas une grandeur intrinsèque ni conservatrice; elle varie en fonction de son environnement. Autrement dit, le comportement d'un segment de dislocation à un instant donné dépend du comportement des autres segments à ce même instant. Il s'agit là donc d'un système différentiel couplé typique impossible à résoudre analytiquement sauf dans les cas d'extrême simplicité. L'approche énergitique, appelée modèle de tension de ligne [8], est, par conséquent, limitée et ne s'applique qu'aux cas où l'interaction entre segments de dislocations reste négligeable ou constante.

L'idée originale, à la base des simulations DD, est de recourir aux formules connues de champs de contraintes engendrés par des segments infinitésimaux de dislocations [9, 10]. Il suffit donc de 

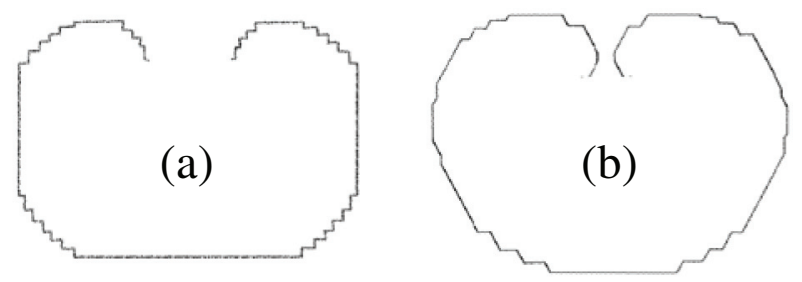

Figure 3. Comparaison entre les résultas de le DM avec ceux de la DD après l'adoption d'une loi de transition d'échelle.

discrétiser les lignes de dislocations en un grand nombre de segments pour connaître la force effective locale en n'importe quel point de la dislocation. Ensuite, si une loi de mobilité est connue pour le matériau, c'est-à-dire la relation entre la vitesse du segment et la force effective locale, il est possible de prédire le déplacement du segment dans le pas de temps relatif à une itération de simulations. L'exigence fondamentale à respecter dans ce type de simulations est d'assurer que le pas de temps est suffisamment petit pour ne pas s'écarter irréversiblement de l'équilibre local quasi-statique. Bien évidemment, le processus de discrétisation constitue un aspect clé des simulations DD. Dans la Figure 3, un exemple de source de Frank-Read activée par la contrainte appliquée avec deux procédures de discrétisation.

Tous les codes de simulations DD sont basés sur le principe décrit ci-haut (avec une grande variance concernant le processus de discrétisation).

Mais, l'approche «force » utilisée dans la DD ne permet pas de rendre compte des réactions entraînant une modification de la structure du cœur comme les jonctions ou l'annihilation. Afin de reproduire les microstructures expérimentales, il faut donc introduire des règles locales imposant le comportement particulier relatif à chaque type de réaction. Ainsi, avec le niveau actuel de maturité du code, tous les mécanismes de la plasticité cristalline peuvent être explorer et analysés quantitativement sans aucune variable ajustée.

Dans la suite, une description du fonctionnement du code $\mathrm{mM}$ permet de mettre en évidence les principales étapes d'une simulation DD typique.

\subsection{Fonctionnement du code}

Avant de démarrer les simulations, il faut bien évidemment définir une configuration initiale de segments. Le plus souvent, il s'agit d'une distribution aléatoire de sources de Frank-Read sur tous les systèmes de glissement. Cette configuration est déterminée en fonction de l'application souhaitée et générée par une procédure indépendante. Au lancement du code $\mathrm{mM}$ deux blocs principaux sont exécutés.

\subsubsection{Bloc d'initiation}

Il s'agit de l'ensemble de procédures dédiées à la lecture des fichiers de données, à la tabulation des principales propriétés topologiques ainsi que les règles locales d'interaction. Le comportement du code est entièrement déterminé par les valeurs numériques assignées aux variables globales dans cette partie. Les étapes importantes de ce bloc sont :

- Charger la Base de Vecteurs de Discrétisation (BVD) en fonction de la structure cristallographique considérée. Il s'agit d'un ensemble de vecteurs entiers 3D représentant les directions de discrétisation permises pour tous les systèmes de glissement. Dans la version ancienne du code (version Coin-Vis), deux vecteurs étaient considérés par système : un parallèle au vecteur de Burgers et un orthogonal (direction coin). Dans le code Vis-Coin, il est impossible de décrire proprement les processus de formation ou destruction de jonctions, car les directions des jonctions ne sont pas explicitement 
présentes dans la BVD. En effet, ces directions ne correspondent pas forcément aux directions coin et vis. La version actuelle du code $\mathrm{mM}$ intègre explicitement les directions d'intersection des plans de glissement, assurant ainsi un traitement transparent des jonctions. L'influence du nombre de vecteurs de discrétisation est également visible sur la prise en compte de la vraie courbure de la dislocation, voir Figure 3.

- Optimiser l'unité de longueur de l'espace de simulation, $A$, en fonction de la longueur de discrétisation imposée. L'avantage principal de la DD discrète est de pouvoir manipuler des variables entières, comme la position des segments, leurs longueurs et leurs déplacements. Le lien avec l'espace physique est assuré par cette unité : les valeurs entières du code sont en réalité des multiples de $A$.

- Sélectionner les conditions aux limites, éventuellement établir des Conditions Périodiques aux Limites (CPL). Il s'agit, en effet, d'un aspect important et délicat des simulations [7]. Si les CPL ne sont pas appliquées, les surfaces de la boîte de simulation peuvent être considérées comme des surfaces hermétiques ou libres. Dans le dernier cas, il faut associer les forces images appliquées aux dislocations. Dans ce cas, le volume dans lequel les dislocations ont un comportement volumique se réduit sensiblement par rapport à la taille de la boîte $(\approx 10 \mu \mathrm{m})^{3}$. Opter pour les surfaces libres conduit donc à restreindre l'utilisation de la DD à l'exploration des phénomènes locaux de la plasticité (interactions individuelles, etc. ... ). En revanche, une introduction judicieuse des CPL plonge la boîte de simulation dans un milieu élastique infini représentatif d'un monocristal.

\subsubsection{Boucle principale}

Une fois les variables et tableaux globaux renseignées, le code entre dans la boucle principale sur le temps. A chaque itération, les différentes étapes suivantes sont exécutées.

- En fonction du chargement (vitesse ou contrainte imposée, fatigue, relaxation, etc...), la contrainte est incrémentée et le tenseur de la contrainte appliquée est mis à jour.

- Les règles topologiques sont vérifiées pour tous les segments. Les segments sont, par exemple, découpés en trois lorsque leurs tailles dépassent le double de la longueur de discrétisation souhaitée.

- On calcule les trois composantes de la force sur chaque segment [10] : (i) force appliquée de Peach et Koehler ; (ii) force interne induite par les autres segments et la tension de ligne [9] tenant compte de la courbure locale. La résultante de ces forces est appelée la force effective.

- Une loi de mobilité est utilisée pour calculer la vitesse des segments en fonction de la force effective. L'incrément de temps étant constant, nous pouvons ainsi évaluer le déplacement à réaliser par chaque segment.

- Ensuite, les segments sont explicitement déplacés avec l'amplitude prévue dans la limite de compatibilité topologique. Si, par ailleurs, un obstacle est détecté dans le trapèze de vol, le segment est arrêté sur l'obstacle et un traitement de contact est engagé. Selon les règles locales, rendant compte des réactions de cœur des dislocations et établies dans le bloc initial, une rotule (segment de taille nulle) est introduite au point d'intersection des deux segments. Dans les itérations suivantes et en fonction du champ de forces locales, cette rotule peut se développer pour initier la réaction (jonction, annihilation) ou disparaître lorsque le bilan énergétique est défavorable. Dans ce cas les segments poursuivent leurs chemins sans réaction.

A la fin de la boucle, les grandeurs mésoscopiques sont calculées : déformation plastique totale, densité de dislocations, etc. Ce sont ces grandeurs qui sont utilisées pour incrémenter la contrainte appliquée afin de respecter le mode de chargement imposé.

\subsection{Exemple de base de discrétisation}

Nous considérons ici l'exemple de la structure CC. De part sa spécificité, la démarche adoptée pour générer la BVD met en évidence les conditions générales à respecter. 

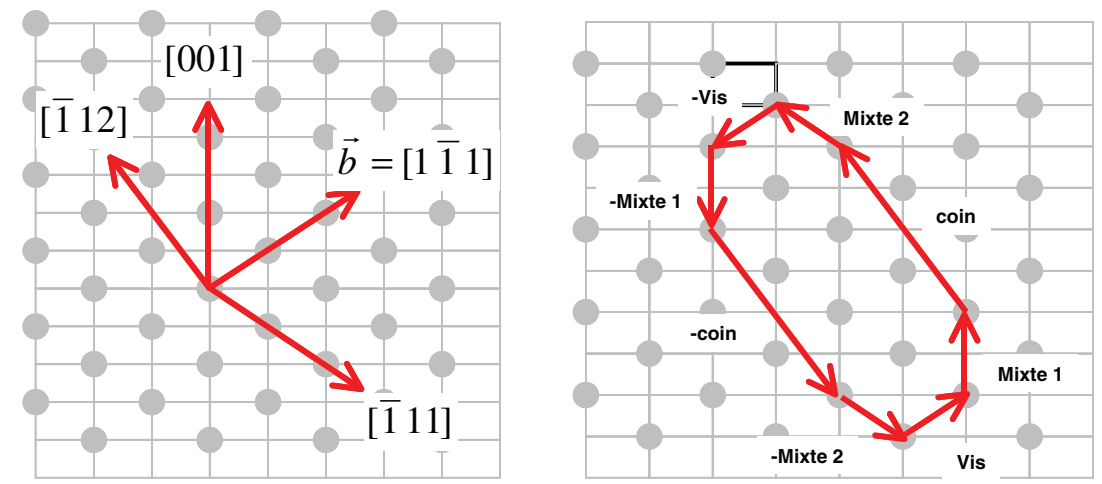

Figure 4. Activation sous contrainte d'une source de Frank-Read, (a) discrétisation selon deux vecteurs (vis et coin) ; (b) discrétisation selon quatre vecteurs (vis, coin, et deux mixtes).

Les positions atomiques dans un plan $\{110\}$ représentent une anomalie par rapport aux structures CFC et HC. En effet, les directions cristallographiques (coin et vis) de type $\langle 112\rangle$ et $\langle 111\rangle$ ne constituent pas d'axe de symétrie dans le plan de glissement, voir figure 4. Par ailleurs, les directions d'intersections entre plans de type $\{110\}$ ne sont pas équivalentes. Elles sont de nature $\langle 100\rangle$ et $\langle 111\rangle$. Le nombre de directions de discrétisation par système étant fixé à 4 , ces directions doivent représenter : une direction

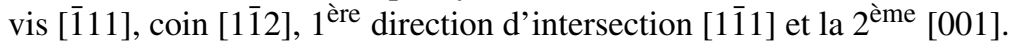

Les directions mixtes ne font pas le même angle avec la direction du vecteur de Burgers et, donc, ne sont pas de même caractère. Ceci conduit inévitablement à une configuration asymétrique des boucles de dislocations, voir Figure 4.

Cette asymétrie rend également difficile la définition des vecteurs de déplacement associé à chaque segment. Il s'agit de vecteurs perpendiculaires aux directions de discrétisation, voir Figure 4, et dont les normes doivent correspondre à des translations entières du réseau, compatibles avec les vecteurs voisins. Comme ceux-ci sont différents en norme et en nature, il faut relaxer un certain nombre de conditions de discrétisation qui ont été adoptée pour les structures CFC et HC.

Comme exemple, nous donnons une solution de la BVD correspondant au système [111](101) et qui vérifie toutes les conditions citées ci-dessus. Cette base n'est pas unique, mais elle représente la solution minimisant l'ensemble des normes de vecteurs de déplacement.

$$
\begin{aligned}
& \left.\mathrm{V}=\text { vecteur ligne : [ } \begin{array}{lll}
1 & -1 & 1
\end{array}\right] \text {, vecteur dplacement : [4 } 8 \text { 4] }
\end{aligned}
$$

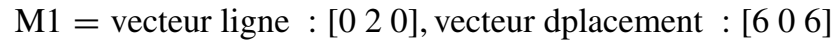

$$
\begin{aligned}
& \mathrm{C}=\text { vecteur ligne : [ } \begin{array}{ll}
2 & 4
\end{array} 2 \text {, vecteur dplacement : [2 -2 2] } \\
& \text { M2 = vecteur ligne : [ } \left.\left.\begin{array}{lll}
1 & 1 & 1
\end{array}\right] \text {, vecteur dplacement : [ } 4 \begin{array}{ll}
4 & -8
\end{array}\right] \text {. }
\end{aligned}
$$

\subsection{Validation du code}

Afin de s'assurer que cette base asymétrique n'entraîne aucune modification du comportement des dislocations à l'échelle mésoscopique, une étape de validations doit être réalisée.

Un des paramètres le plus important à tester est la longueur de discrétisation, $\lambda$. Ce dernier, bien évidemment, ne correspond pas à une grandeur physique. Sa valeur, néanmoins, détermine tout le comportement du code puisqu'elle fixe la précision de la description de la courbure des dislocations. D'un autre côté, le nombre de segments, à microstructure égale, est proportionnel à $\lambda$. Le choix de la valeur de $\lambda$ fait donc l'objet d'un compromis délicat entre précision et efficacité numérique. 


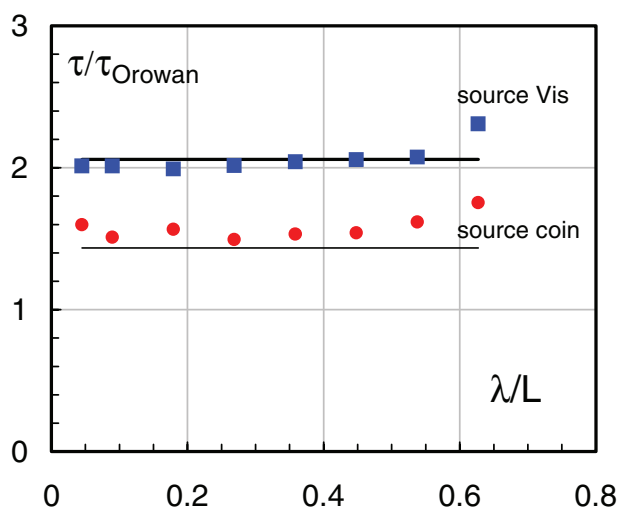

Figure 5. À gauche, positions atomiques dans un plan $\{110\}$ de la structure $\mathrm{CC}$ et les quatre directions imposées par la physique du glissement et d'interaction entre systèmes de glissement. A droite : base asymétrique de vecteurs de discrétisation dans le plan (110). On peut noter que les vecteurs mixte1 et mixte2 ne font pas le même angle avec la direction vis.

La méthode généralement utilisée pour déterminer le domaine de validité pour $\lambda$ est de mesurer la contrainte critique de fonctionnement des sources de Frank-Read (FR). L'exemple des sources FR est récurent car il constitue un des rares cas où la contrainte critique a pu être identifiée en simulations statiques [11] ainsi qu'en approche analytique [8]. Cette contrainte peut être écrite sous la forme :

$$
\tau_{c}=B \frac{\mu b}{L} \ln \frac{L}{b}+C,
$$

où $B$ est une constante dépendante du caractère initial de la source, $\mu$ le module de cisaillement, $b$ le vecteur de Burgers, $L$ la longueur de la source et $C$ une constante dépendante de la position de la nature des bras de la source.

Il s'agit, donc, de déterminer le domaine de $\lambda$, où la contrainte critique mesurée reste proche de la contrainte théorique, voir Figure 5. La contrainte critique ne doit pas dépendre de la longueur de discrétisation. Dans la Figure 5, nous pouvons constater que pour une longueur de discrétisation comprise entre $0,1 \mathrm{~L}$ et $0,5 \mathrm{~L}$, la contrainte critique mesurée en simulation DD correspond à la contrainte théorique donnée par Foreman [9]. Pour les petites valeurs de $\lambda$, la contrainte critique mesurée augmente, phénomène appelé dans notre jargon « durcissement informatique ».

\subsection{Application : simulation DD du durcissement par précipitation}

Le durcissement par précipitation représente le principal processus métallurgique de durcissement des matériaux [13]. Lorsque les précipités sont petits et cohérents, les précipités peuvent être cisaillés. Dans tous les autres cas, ils constituent des zones impénétrables par les dislocations (mécanisme d'Orowan), augmentant ainsi fortement la limite d'écoulement. Cependant, la modélisation du durcissement par précipitation reste encore très difficile en raison de deux effets : la distribution et la taille finie des précipités. Or, il s'agit d'un mécanisme tout à fait à la portée des simulations DD, où le durcissement peut être évalué sans hypothèse préalable. Le cas du durcissement d'Orowan a déjà fait l'objet de travaux en DD [4]. Ici, nous traitons le même exemple abordé dans la DM, i.e. durcissement par cavités.

La cavité est représentée dans l'espace de simulation par une sphère résistante $\left(\tau_{\text {shear }}\right)$ au passage de la dislocation. Cette dernière se courbe alors de part et d'autre de la sphère ce qui provoque une augmentation considérable de la contrainte effective $\left(\tau_{\text {eff }}\right)$ sur le ou les segments bloqués par la sphère. $\mathrm{Si}$, à un certain moment, $\tau_{\text {eff }}$ dépasse $\tau_{\text {shear }}$, le segment est autorisé à pénétrer la sphère, ce qui équivaut au cisaillement du précipité à l'échelle atomique. 


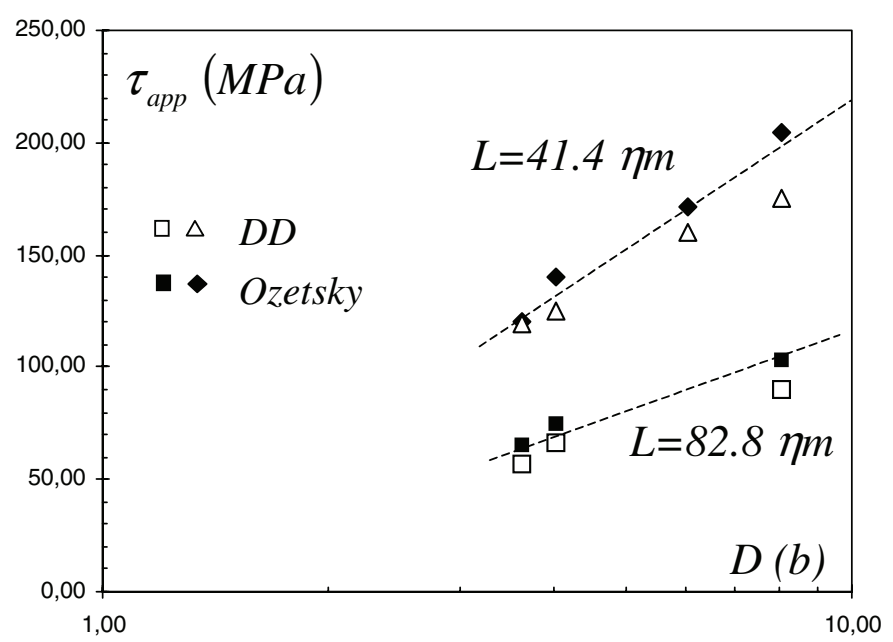

Figure 6. Influence de la longueur de discrétisation normalisée par la longueur de la source sur la contrainte critique normalisée par la contrainte d'Orowan [12] $(\mu \mathrm{b} / L)$. Comparaison entre simulation statique [11] et DD.

Afin de vérifier et valider notre approche multiéchelle, c-à-d le passage de l'échelle atomique à l'échelle continue à travers l'estimation de $\tau_{\text {shear }}$, nous avons comparé avec les résultats de la DM, voir Fig 5. Comme le montre la figure, l'accord est très bon pour les faibles tailles de cavité, même-si la prédiction de la DD est systématiquement inférieure à celle de la DM et de plus en plus marqué pour les grandes tailles. Deux raisons sont derrière cette tendance. D'abord, la configuration de la dislocation à la contrainte maximale ne correspond pas à une résistance sur tout le diamètre de la cavité. Deuxièmement, pour les cavités de grandes tailles, l'arrachement de la dislocation se fait après génération de dipôles de dislocation vis rectilignes au bord de la cavité. Ce n'est donc plus la tension de ligne qui contrôle le cisaillement mais la mobilité des dislocations vis : la loi de transition introduite ci-haut n'est pas valable pour les grandes cavités ou les fortes obstacle.

Fig. 6 représente, par conséquent, une double validation : celle de la loi d'échelle et une validation ultime de la méthode de simulation DD. Il est intéressant de signaler ici que, actuellement, concernant le problème de cisaillement de cavités, nous nous ne disposons pas de modèle permettant de prédire la résistance mécanique de ce type d'obstacles. Il est donc nécessaire d'utiliser la DD afin de prédire le durcissement correspondant.

Les résultats montrés dans Fig. 6 montre la contrainte critique d'une rangée périodique infinie de précités (configuration obtenue du fait de l'application des conditions périodiques aux limite dans la $\mathrm{DM}$ et la $\mathrm{DD}$ ). Le cas d'une distribution aléatoire de cavités est tout à fait possible dans la boîte de simulation de DD. Le durcissement correspondant est inférieur à celui des rangés périodiques du fait de la fluctuation de l'espacement entre cavités.

\section{CONCLUSIONS}

Le « monde » des dislocations s'étale sur plusieurs échelles; chacune est indispensable pour compléter la description du comportement.

L'exemple du durcissement par cavités montre toute la puissance de la démarche multiéchelle. Alors que le caractère atomique de l'interaction est fondamental pour la détermination de la force de l'interaction, le durcissement reste à l'échelle macroscopique. Sans cette démarche, il est impossible d'aboutire à une description sur une base physique d'une propriété concernant les dislocations. 
Les deux prochains défi multiéchelles sont clairement associés à deux transitions :

- La traduction vers les échelles supérieures des détails atomiques de l'activation thermique représente le seul obstacle à l'utilisation par la DD de tous les résultats de la DM ;

- La prise en compte des hétérogénéités de déformation inhérentes aux caractère discret de du glissement plastique reste très insuffisant. Par exemple, l'apparition des bandes de glissement ou la concentration de contraintes/déformation dans le voisinage des joints de grain nécessite l'introduction d'une variable spatiale locale dans les codes d'EF.

Ici, les difficultés derrière ces défis ne sont pas d'ordre numérique ou de puissance de calcul. Il s'agit de véritables lacunes dans notre compréhension du comportement mécanique dues au fait que, précisément, ces phénomènes interviennent aux frontières des domaines bien établis.

Lorsque ces transitions sont établies, toute la chaîne du comportement mécanique des matéiaux sera identifiée et caractérisée sans variable ajustée.

\section{Références}

[1] Hirth J.P. and Lothe L., In Theory of Dislocations, $2^{\text {nd }}$ Edition, Wiley-Inter-Science, New York (1982).

[2] Osetsky Yu.N. and Bacon D.J., Modelling Simul. Mater. Sci. Eng. 11 (2003) 427.

[3] Ackland G.J., Bacon D.J., Calder A.F. and Harry T., Phil. Mag. A 75 (1997) 713.

[4] Monnet G., Phil. Mag. 86 (2006) 5927.

[5] Devincre B., dans « Computer Simulation in Materials Science. Amsterdam, North-Holland », Kirchner H.O., Pontikis V. and Kubin L.P., Eds.: Kluwer Academic Publishers ; p. 309 (1996).

[6] Devincre B., Kubin L.P., Lemarchand C. and Madec R., Mater. Sci. Eng. A 211 (2001) 309.

[7] Monnet, Devincre B. et Kubin L., Acta Mater. 52 (2004) 4317.

[8] De Wit G. et Koehler J.S., Phys. Rev. 116 (1959) 1113.

[9] Foreman A.J.E., Phil. Mag. 15 (1967) 1011.

[10] Devincre B. and Condat M., Acta Met. Mat. 40 (1992) 2629.

[11] Foreman A.J.E., Phil. Mag. 15 (1967) 441.

[12] Orowan E., Symposium on Internal Stresses in Metals and Alloays, London : The Institute of Metals ; p. 451 (1948).

[13] Mott N.F. and Nabarro F.R.N., Proc. Phys. Soc. 52 (1940) 86. 
